\title{
Los desafíos de la integración en el País Vasco: una perspectiva psicosocial
}

\author{
Miguel Ángel Navarro Lashayas \\ SOS Racismo \\ $<$ m.lashayas@gmail.com>
}

\begin{abstract}
Gizarteratze hitza da migratzaileei buruz eta talde horren inguruko politikei buruz hitz egitean gehien erabiltzen den hitzetako bat. Immigrazioaren inguruko hainbat plan gauzatu dira Euskadin, erakundeek bideratuta, atzerritarrak euskal gizartean txertatzen laguntzeko helburuarekin. Akademiatik gero eta tresna gehiago sortzen dira kontzeptu hori ikuspegi ahalik eta objektiboenetik neurtzeko asmoz. Hala ere, gizarteratzea baliabide ekonomikoak, lan bat edo etxebizitza bat edukitzea baino askoz ere gehiago da; zerikusia du, halaber, beste hainbat gairekin, nola diren pertsonen ongizate psikologikoa eta soziala, zehazki herritartasuna lortzeko aukera, akulturazioaren estresa, parte-hartze soziala eta politikoa, gizartesareak, bereizkeria, arrazismoa eta xenofobia edo gizarte-bazterketa. Artikuluak gai horiek guztiak berrikusten ditu, Euskal Herrian zentratuz.
\end{abstract}

\section{GAKO-HITZAK:}

Psikologia soziala, immigrazioa, gizarteratzea, gizarte-politikak, bereizkeria.
La palabra integración es una de las más utilizadas cuando se hace referencia a las personas migrantes y las políticas relativas a dicho colectivo. En Euskadi, se han desarrollado diversos planes institucionales de inmigración, cuyo objetivo era contribuir a la integración en la sociedad vasca de las personas extranjeras. Desde la academia, cada vez se construyen más instrumentos que tratan de medir dicho concepto desde una perspectiva lo más objetiva posible. Sin embargo, la integración es mucho más que disponer de recursos económicos, un trabajo o una vivienda; también tiene que ver con cuestiones como el bienestar psicológico y social de las personas, concretado en aspectos como el acceso a la ciudadanía, el estrés de aculturación, la participación social y política, las redes sociales, la discriminación, el racismo y la xenofobia o la exclusión social. El artículo repasa estos temas, centrándose en el País Vasco.

\section{Palabras Clave:}

Psicología social, inmigración, integración, políticas sociales, discriminación. 


\section{Introducción}

Cuando se habla de inmigración, se emplean muchos términos que han sido asumidos socialmente, pero que en ocasiones están vacíos de contenido en su utilización, y dista mucho de haber un consenso en cuanto a su definición. Ejemplo de ello pueden ser conceptos como interculturalidad, cultura, racismo, ciudadanía, etnicidad o integración. Este último es, probablemente, el término más utilizado en el campo de la política social de inmigración, pero también en ámbito ciudadano y académico. ¿Se refieren todos a lo mismo? Cuando una persona argumenta que 'los inmigrantes no se integran en nuestra sociedad', ¿está concibiendo la integración de la misma manera que un político o que un estudioso de las migraciones? Probablemente no; el término integración se utiliza muchas veces como un sustituto de asimilación, y cuando se habla de integración, en realidad se hace referencia a una asimilación encubierta, esto es, 'que sean como nosotros'. El refranero español es rico y también en esto tiene su propia fórmula: 'allá donde fueres, haz lo que vieres'.

Sin embargo, la integración no es eso; en ningún caso debería suponer un abandono forzado de cultura e identidades propias. El problema viene al intentar delimitar lo que es. Lorenzo Cachón (2008) habla de los dilemas teóricos de la integración, resumidos en si la integración es una cuestión individual o colectiva (de grupos sociales); si hay que analizar lo objetivo (condiciones de vida) o lo subjetivo (percepciones, sentimientos de pertenencia); si es un proceso o un resultado; si afecta sólo a los inmigrantes o a toda la sociedad. Una definición básica, pero válida, es la que concibe la integración como "el proceso a través del cual uno se convierte en una parte aceptada de la sociedad" (Penninx y Martiniello, 2006: 127, cit. en Cachón, 2008). Otras definiciones intentan avanzar en el concepto, no vinculándolo exclusivamente a la aceptación social e incluyendo la cuestión de la ciudadanía política. Así, podemos definir la integración como "un proceso de creación o generación de nueva ciudadanía; nueva ciudadanía en un doble sentido: por cuanto que los foráneos se incorporan como tales, y por cuanto que se enriquece el concepto de ciudadanía, no excluyendo a nadie ni creando compartimentos de distintos tipos de ciudadanos” (Malgesini y Giménez, 2000).

El Gobierno Vasco no ha sido ajeno a las cuestiones que tienen que ver con la integración de los inmigrantes, y a lo largo de estos años ha creado tres planes de inmigración. En los dos primeros, se remarca la importancia del concepto de ciudadanía inclusiva, a través del reconocimiento de derechos, la asunción de deberes y la inclusión en la comunidad política receptora como medios para la integración. Para ello, se señalan una serie de áreas sobre las cuales es necesario actuar: vivienda, educación, empleo, participación política o sensibilización. El III Plan de Inmigración ofrece algunos cambios sustanciales, poniendo el acento en la basculación de la acogida hacia la integración, la convivencia, la gestión de la diversidad y el trabajo por la igualdad y la no discriminación. Para ello, se pasa de las áreas de intervención a las líneas estratégicas: la estructuración y articulación intra e interinstitucional, el acceso al empleo, la formación y la educación, la gestión de la diversidad desde lo público, la participación, la convivencia y la lucha contra la discriminación.

Este tipo de enfoque es indispensable. Sin embargo, tiene sus limitaciones, al relegar a un segundo plano el papel de la persona migrante para decidir sobre su propio proceso de incorporación, y no tener en cuenta la visión subjetiva de los individuos objeto de las políticas, así como otros aspectos psicosociales que pueden ser claves para avanzar en los procesos de integración social.

\section{La medición de la integración}

La investigación acerca de los procesos de integración que ocurren en las sociedades receptoras de personas migrantes se ha centrado fundamentalmente en datos de encuestas existentes o creadas ad hoc, estadísticas oficiales y medidas legislativas. Buen ejemplo de ello es el Índice de Políticas de Integración de Inmigrantes (MIPEX), coordinado por el Barcelona Centre for International Affairs (CIDOB) y el Migration Policy Group (MPG), un indicador sintético construido mediante siete variables:

1. Acceso de los inmigrantes al mercado de trabajo, que mide la inclusión y exclusión de los inmigrantes de determinados puestos de trabajo, el ajuste del flujo de inmigrantes a la oferta de empleo, las facilidades para la obtención del permiso de trabajo y el acceso a los derechos sociales.

2. La reunificación familiar, que mide las facilidades y requisitos para hacerla afectiva.

3. La educación, que mide las facilidades de acceso a la educación pública, la enseñanza del idioma o el enfoque intercultural, entre otras cuestiones.

4. Las facilidades para la obtención del permiso de residencia a largo plazo, que mide particularmente el tiempo mínimo requerido para su concesión.

5. La participación política, que mide el derecho de asociación política y sindical, así como la participación como representante y votante.

6. El acceso a la nacionalidad, variable relativa a las exigencias de estancia temporal y los requisitos para obtener la ciudadanía.

7. Las medidas antidiscriminatorias, que miden la discriminación por motivos étnicos, raciales o religiosos, y las posibilidades de reclamar medidas de protección contra la discriminación.

La literatura internacional apoya este enfoque y subraya la importancia de algunos factores en la integración de los y las inmigrantes, tales como el acceso al empleo, la vivienda o los servicios básicos en 
situación de igualdad, así como el reconocimiento de los derechos políticos y de participación ciudadana (Cachón, 2009; De Lucas y Solanes, 2009).

Recientemente se ha publicado un estudio (Godenau et al., 2014) realizado por los diferentes observatorios de la inmigración de España con la finalidad de ofrecer un modelo de medición del proceso de integración de los extranjeros en la sociedad, en sus distintas dimensiones. Este modelo se basa en un sistema de indicadores amplio y variado, fundamentado en cuatro dimensiones: empleo, bienestar, relaciones sociales y ciudadanía.

Sin embargo, la necesidad de disponer de datos de fuentes oficiales que permita comparar diferentes regiones del Estado ha limitado considerablemente los indicadores que se utilizan. De este modo, para las dos dimensiones más vinculadas a la visión psicosocial, el bienestar y las relaciones sociales, se utilizan indicadores como el número de hogares que llegan con dificultad a final de mes, el gasto medio por hogar, el número medio de personas por habitación, el estado de salud, los estudios superiores, el número de hogares mixtos, la convivencia de la pareja en el hogar, el número de hogares con menores dependientes, la capacidad de comunicarse en español o la actitud de la población hacia las personas extranjeras. Como vemos, son indicadores que, al igual que el MIPEX, analizan lo objetivo, y no lo subjetivo.

\section{3. ¿De qué hablamos al referirnos a la perspectiva psicosocial?}

La perspectiva psicosocial pretende estudiar las relaciones entre algunas manifestaciones de la salud y determinados componentes del ordenamiento social, aunque sin el determinismo que defiende la prevalencia de lo social sobre lo individual, acercándonos a los sentimientos, creencias y experiencias que las personas tienen respecto a determinados aspectos del orden, la realidad y de las relaciones sociales. Desde esta mirada, el orden social no sólo ofrece datos susceptibles de convertirse en estadísticas; también está en la mente de cada uno de nosotros. Eso nos permite hablar de algunos de los componentes del orden social, no tal y como propiamente son, sino tal y como son vividos, experimentados y percibidos por los sujetos (Blanco y Díaz, 2006). De esta manera, el sentimiento de integración, de confianza en los otros y en la sociedad, de autoeficacia y capacidad de control, de utilidad social y de sentido de nuestra vida reflejan las características objetivas de la estructura y ordenamiento social. Ignacio Martín Baró (2003: 338) lo reflejó muy bien cuando afirmó que había que ver la salud "no desde dentro afuera, sino de afuera adentro; no como la emanación de un funcionamiento individual interno, sino como la materialización en una persona del carácter humanizador 0 alienante de un entramado de relaciones sociales".
El concepto que mejor refleja al sujeto sociohistórico del que habla la perspectiva psicosocial, inserto en una red de relaciones interpersonales e intergrupales contextualizadas en una sociedad concreta, es el de bienestar. Albert Einstein (cit. en Blanco y Rodríguez Marín, 2007) dijo que "la preocupación por el bienestar del ser humano por su destino debe ser el principal motivo de toda empresa técnica, a fin de que las creaciones de nuestra inteligencia sean beneficiosas y no una maldición para la humanidad. No olvidéis jamás eso en medio de vuestros gráficos y vuestras ecuaciones". La Organización Mundial de la Salud ya lo advirtió en su asamblea fundacional (1948) cuando expuso que "la salud es un estado de bienestar completo, físico, social y psicológico, y no solamente la ausencia de enfermedad o de invalidez" La filosofía que sustenta el concepto de bienestar apunta a un modelo de intervención que, al mirar el contexto social donde está inserto el sujeto, se ve obligado a centrar su atención en el cambio social, en la variación de aquellas condiciones externas que afectan al bienestar de las personas.

Las dimensiones del bienestar abarcan el bienestar subjetivo (evaluación global del individuo), el bienestar psicológico (sentirse bien consigo mismo, tener relaciones positivas con los demás, autodeterminación, dominio del entorno, sentido de vida y posibilidad de desarrollo de las potencialidades) y el bienestar social, entendido éste como “la valoración que hacemos de las circunstancias y el funcionamiento dentro de la sociedad" (Keyes, 1998).

\section{4. ¿Por qué incorporar la perspectiva psicosocial en el estudio de la integración?}

Explorar el bienestar de las personas migrantes nos aporta una visión más global e integral de cómo se producen los procesos de integración y de qué factores pueden influir en ellos. Se trata de complementar los indicadores exclusivamente objetivos o jurídicos para medir los procesos de integración, asunto que cada vez es más cuestionado (De la Mata et al., 2010; Spencer y Cooper, 2007). Esta visión se alinea con lo que se conoce como los métodos de investigación mixtos, que combinan lo cuantitativo con lo cualitativo, incorporando las experiencias y vivencias subjetivas y su interrelación con los datos objetivos (Tashakkori y Teddlie, 2010). En este sentido, variables como la discriminación percibida o el sentimiento de integración, de pertenencia o de control de la situación son ejemplos de indicadores subjetivos de gran validez cuando hablamos de integración.

Las dimensiones del bienestar que hemos visto en el apartado anterior pueden ser una buena guía para analizar los procesos de integración. Las dimensiones y sus componentes se expresan en el Cuadro 1. 


\begin{tabular}{|c|c|c|}
\hline \multicolumn{3}{|c|}{ Cuadro 1. Dimensiones y componentes del bienestar } \\
\hline Bienestar subjetivo & Bienestar psicológico & Bienestar social \\
\hline $\begin{array}{l}\text { 1. Satisfacción: juicio o evaluación global } \\
\text { de los diversos aspectos que una per- } \\
\text { sona considera importantes en su vida. } \\
\text { 2. Afecto positivo: resultado de una } \\
\text { experiencia emocional placentera ante } \\
\text { determinada situación vital. } \\
\text { 3. Afecto negativo: resultado de una } \\
\text { experiencia emocional negativa ante } \\
\text { una determinada situación vital. }\end{array}$ & $\begin{array}{l}\text { 1. Autoaceptación: sentirse bien consigo } \\
\text { mismo, tener actitudes positivas hacia uno } \\
\text { mismo. } \\
\text { 2. Relaciones positivas con los otros: } \\
\text { mantenimiento de relaciones estables y } \\
\text { confiables. } \\
\text { 3. Autonomía: capacidad para mantener las } \\
\text { propias convicciones (autodeterminación), } \\
\text { la independencia y la autoridad personal. } \\
\text { 4. Dominio del entorno: habilidad personal } \\
\text { para elegir o crear entornos favorables } \\
\text { para satisfacer deseos y necesidades } \\
\text { propias. } \\
\text { 5. Objetivos vitales que permitan dar sentido } \\
\text { a la vida. } \\
\text { 6. Crecimiento personal: empeño por } \\
\text { desarrollar las potencialidades y seguir } \\
\text { creciendo como persona. }\end{array}$ & $\begin{array}{l}\text { 1. Integración social: sentimiento de perte- } \\
\text { nencia, establecimiento de lazos sociales. } \\
\text { 2. Aceptación social: confianza en los otros } \\
\text { y aceptación de los aspectos positivos y } \\
\text { negativos de nuestra propia vida. } \\
\text { 3. Contribución social: sentimiento de utili- } \\
\text { dad, de ser capaces de aportar algo a la } \\
\text { sociedad en que vivimos. } \\
\text { 4. Actualización social: confianza en el futuro } \\
\text { de la sociedad, en su capacidad para } \\
\text { producir condiciones que favorezcan el } \\
\text { bienestar. } \\
\text { 5. Coherencia social: confianza en la capa- } \\
\text { cidad para comprender la dinámica y el } \\
\text { funcionamiento del mundo en el que nos } \\
\text { ha tocado vivir. }\end{array}$ \\
\hline
\end{tabular}

Fuente: Blanco y Díaz (2006).

Está claro que el bienestar no afecta sólo a la población migrante, sino a toda la población, que puede obtener unos malos resultados en este aspecto, pero es cierto que muchos de los componentes del bienestar social nos permiten situar a la persona en relación con el entorno en el que está inserta y, por lo tanto, son un buen indicador de cómo está viviendo y sintiendo la sociedad 'de acogida'.

Por otro lado, las migraciones, tienen un importante componente estructural, cultural, social y familiar. Gran parte del sentido que encuentra la persona migrante respecto a lo que le ocurre en el país de acogida está basado en su ideología y experiencias previas, pero también en lo que habla y comparte con otros, generalmente compatriotas o personas que están pasando por su misma situación. Además, el efecto de la migración en la persona, sus comportamientos, emociones y pensamientos sólo puede entenderse en su contexto.

En definitiva, debemos considerar distintos niveles de intervención (micro, meso, macro) [Bronfenbrenner, 1979] para facilitar la integración de las personas. Por ejemplo, los cambios en la legislación sobre reagrupación familiar (nivel macro) pueden producir cambios en la estructura familiar (nivel meso), que afecta, en última instancia, al desarrollo del locus de control, la autoeficacia y a las estrategias de afrontamiento tanto de las personas en la sociedad de origen como en la de acogida (nivel micro). Es decir, los diferentes niveles guardan una lógica, ningún cambio en alguno de ellos por sí sólo consigue resultados si no se ve acompasado por cambios en la misma dirección en el resto.

\section{5. ¿Cuáles son los retos futuros para la integración en el País Vasco y qué aporta a ellos la perspectiva psicosocial?}

En los planes de inmigración del País Vasco, ha habido una evolución desde medidas encaminadas a favorecer la acogida e integración, y a conseguir estructurar un entramado público capaz de liderar dichos procesos, hacia medidas donde la responsabilidad es compartida entre la sociedad de acogida y las personas migrantes, pasándose a hablar de ciudadanía y convivencia intercultural. A pesar de que en los dos primeros planes se definía la integración como "un concepto bidireccional, que implica un proceso dinámico de adaptación recíproca entre la población inmigrada y la población originaria” que "implica la posibilidad de desarrollo libre de las diversas identidades en pie de igualdad" y es "incompatible con la existencia de la categoría jurídica de irregularidad”, lo cierto es que la sociedad de acogida tenía un papel casi de espectador. Un ejemplo es que las palabras ‘discriminación' y ‘racismo' tan sólo aparecen en la revisiones del marco normativo internacional. El último plan de inmigración supone, en parte, superar dicha limitación, pero por el contrario, lima muchas de las críticas anteriores a la Ley de Extranjería.

En cualquier caso, hay una serie de cuestiones que se han tratado con mayor o menor profusión en los planes de inmigración y que resultan claves a la hora de definir un modelo de política de integración en el País Vasco. De las respuesta que se les dé dependerá, en gran medida, su éxito y, por lo tanto, la convivencia y cohesión social. Pasemos a analizarlas.

\subsection{Acceso a la ciudadanía}

El acceso a la ciudadanía hace referencia al reconocimiento de derechos plenos hacia las personas migrantes. El I Plan de Inmigración habla de ciudadanía inclusiva, desligando dicho concepto del componente nacional. Apuesta por un estatuto uniforme para todos sus titulares, que equipare a las personas en el ámbito mínimo de los derechos humanos necesarios: derechos civiles, políticos, económicos, sociales y culturales. Parece que, con el paso de los años, ha ido perdiendo fuerza esta visión y hemos asistido a la pérdida progresiva de la condición de 
igualdad de derechos para todas las personas. Algunas medidas que apuntan esta dirección son:

- Los cambios en la normativa de acceso a la renta de garantía de ingresos (Ley 18/2008, de 23 de diciembre regulada por el Decreto 147/2010, de 25 de mayo) que eleva el tiempo de empadronamiento para su acceso a los tres años (Ley 4/2011, de 24 de noviembre).

- La petición del año de empadronamiento para acceder a la tarjeta individual sanitaria (Decreto 114/2012, de 26 de junio).

- La imposibilidad de acceder a Etxebide para las personas que se encuentran residiendo de manera irregular.

- Restricciones en el acceso a la matriculación en la.Escuela Oficial de Idiomas únicamente a las personas con permiso de residencia o trabajo.

- Modificaciones de normativas municipales, como la realizada en Bilbao para evitar la apertura de centros de culto en edificios de viviendas (Ayuntamiento de Bilbao, 2013).

- Redadas a personas en situación administrativa irregular, como la realizada el 8 de noviembre de 2013 en Zorrozaurre (Bilbao), donde fueron identificadas 54 personas, 36 de ellas detenidas y 11 enviadas al centro de internamiento para extranjeros de Aluche, para ser expulsadas posteriormente.

La desigualdad de acceso a derechos supone:

- Un refuerzo de las dinámicas psicosociales excluyentes, al crear categorías de ciudadanos diferentes dentro de la sociedad.

- Un aumento del malestar y la insatisfacción personal y social, tal y como han mostrado algunas investigaciones que vinculan la percepción de justicia social con el bienestar (Prilleltensky, 2012).

- Un agravamiento de las condiciones sociales en las que la persona migrante se debe insertar.

Este último aspecto es de especial importancia, porque tradicionalmente, al hablar de integración de las personas migrantes, se ponía el foco en el estrés de aculturación (Berry y Kim, 1988; Berry, 2001). El esfuerzo de adaptación, así como las incompatibilidades culturales y los conflictos sociales que surgen durante el proceso de asentamiento y ajuste social son la base del estrés. Las actitudes de aculturación cruzan dos dimensiones, como son la orientación hacia el país de origen y la orientación hacia el país de acogida. Esto conlleva diferentes actitudes hacia la sociedad receptora denominadas integración, asimilación, separación y marginalización. Sin embargo, son numerosos los estudios que vinculan el estrés no tanto al posible choque cultural como a las condiciones de vida en las que se tienen que desenvolver las personas migrantes. Así, por ejemplo, estudios realizados en varios países con solicitantes de asilo político muestran cómo las dificultades para legalizar su situación, los interrogatorios, el aislamiento o el desempleo posteriores a la llegada son mejores predictores de síntomas postraumáticos que las mismas experiencias de detención o tortura en el país de origen (Markez-Alonso, Férnandez-Liria y PérezSales, 2009). En esta misma línea está el concepto del síndrome de Ulises (Achotegui, 2002, 2006), que relaciona el estrés con cuestiones como la soledad, el fracaso migratorio, la lucha por la supervivencia y el miedo, estresores que se ven reforzados por su multiplicidad, cronicidad, intensidad, ausencia de sensación de control y ausencia de red social de apoyo.

Un intento de aunar ambas visiones es aquel que considera la inmigración como estresante en la medida en que es sentida y vivida como tal por la persona. Esta perspectiva agrupa en un mismo modelo tanto el estrés de aculturación como los estresores sociales y desarrolla instrumentos que permiten medir el estrés en la migración (Tomás-Sábado et al., 2007), cuyos componentes son la nostalgia, el choque cultural, la experiencia de discriminación y las condiciones de vida.

En todo caso, tampoco hay que olvidar el papel que la sociedad de acogida tiene en este tipo de procesos vinculados a la aculturación. Así, cada vez se avanza más en poner en valor cuestiones como la competencia cultural de los profesionales que trabajan con personas migrantes, entendiendo ésta como "el conjunto de habilidades académicas e interpersonales que permiten a los individuos aumentar su comprensión y apreciación de las diferencias sociales y culturales en y entre grupos" (Betancourt et al., 2003: 293). Un ejemplo es el Instrumento para la Valoración de la Competencia Intercultural en la Atención en Salud Mental que publicó el Ministerio de Sanidad (Fernández Liria y Pérez-Sales, 2011), dirigido a las instituciones que trabajan en este campo. Sería importante que esta competencia estuviera presente en los planes de formación y gestión del personal de instituciones públicas.

\subsection{Participación social y política}

Estar integrado en el nuevo círculo de vida es un indicador de éxito subjetivo esencial para las personas migrantes. La red de apoyo social cumple funciones como la de apoyo emocional, que ofrece comprensión, afecto y oportunidades de participación social; apoyo informacional y estratégico, con información clave sobre la sociedad de acogida; o apoyo material e instrumental (García Fuster, Herrero Olaizola y Musitu, 1995; Hernández Plaza, Pozo Muñoz y Alonso Morillejo, 2004). Disponer de familiares, amistades y compatriotas en la sociedad de destino puede llegar a tener tanto valor explicativo del proceso migratorio como aquello que es atribuible a las acciones individuales. De esta manera, las acciones que los organismos públicos pongan en marcha para favorecer la reagrupación familiar serán positivas para la integración social. Sin embargo, no hay que olvidar 
que las separaciones familiares por la migración pueden generar un importante malestar, tanto en las personas que han migrado como en las que se han quedado en el país de origen, y sería conveniente crear las estructuras de apoyo necesarias para que la readaptación de roles y funciones de la familia reagrupada no se convierta en un problema.

Por otra parte, las redes sociales no son sólo los lazos directos 0 indirectos que vinculan a unas personas con otras, también aquellos lazos que nos vinculan con la comunidad, con grupos, con instituciones formales o informales y con la estructura social. A pesar del obstáculo que supone la inexistencia del derecho a voto para las personas migrantes, la apertura de espacios de participación social reales es una de las mejores herramientas con las que contamos para promover la integración de las personas y su bienestar (Bess et al., 2009). De esta manera, la intervención debe reconstruir el tejido social, lo que implica actuaciones en los diferentes niveles de análisis (Villasante, 1984):

- Instituciones: administraciones y poderes del Estado, pero además las escuelas, la Iglesia, la Policía, los partidos políticos, el empresariado, los sindicatos y los medios de comunicación, entre otras.

- Sector o grupo formal: grupos formales organizados, como asociaciones de vecinos, asociaciones de padres, madres y alumnos, grupos deportivos o cooperativas.

- Sectores informales: personas y contextos activos, comunicadores de información dentro de la vida cotidiana (bares, mercados, colegios o pandillas)

- Base social: en un principio, no organizada y fragmentada en diferentes perfiles (mujeres, jóvenes, pensionistas, trabajadores o inmigrantes).

Desde la psicología de la liberación, se analiza el fenómeno migratorio en términos de poder y se aboga por la transformación social como medio para alcanzar el bienestar y la justicia social. Para ello, es necesaria la organización comunitaria a través de organizaciones sociales que sean catalizadoras de cambios sociales y promotoras de bienestar colectivo (Paloma y Manzano-Arrondo, 2011).

Tampoco debemos olvidar que conceptos como el de sentido de pertenencia resultan claves a la hora de analizar la integración. El concepto hace referencia a la satisfacción de una persona al sentirse parte integrante de un grupo. El sujeto, de este modo, se siente identificado con el resto de los integrantes, a quienes entiende como pares. El sentido de pertenencia supone el desarrollo de una actitud consciente respecto a otras personas y está íntimamente vinculado a los conceptos de participación, del que acabamos de hablar, y de igualdad, que pasamos a analizar (Alleyne, 2002; Phinney et al., 2001).

\subsection{Discriminación, racismo y xenofobia}

En la relación entre discriminación y psicología, es imprescindible hablar de actitudes. Se distinguen tres componentes en ellas (Eagly y Chaiken, 1993):

- Cognitivo: creencias estereotipadas sobre supuestos rasgos de un grupo.

- Afectivo: evaluación negativa de un grupo, junto con un sentimiento de hostilidad hacia sus miembros.

- Comportamental: tendencia hacia conductas hostiles y de marginación dirigidas a los miembros del grupo.

Sabemos que no siempre existe una relación causal entre los tres componentes: una actitud prejuiciosa (componente afectivo) no necesariamente lleva a un comportamiento congruente con aquélla (discriminación). En gran medida, dependerá de la situación y del contexto social más o menos permisivo con dichas actitudes. De ello se deriva la importancia que tiene crear un clima social favorable hacia las personas migrantes. Las figuras de los prescriptores de opinión (medios de comunicación, partidos políticos) juegan un papel fundamental, por lo que las instituciones públicas deben fomentar una visión positiva de la migración. Alguno de los programas antirrumores que están poniendo en marcha ayuntamientos vascos apunta en esta dirección, y es necesario reforzarlos para ampliar la base de personas a las que se llega con ellos.

Podemos analizar la actitud prejuiciosa de la sociedad vasca ante el fenómeno de la inmigración a través de los barómetros que realiza el Observatorio Vasco de la Inmigración desde hace años, lo que permite hacer comparaciones temporales. El último estudio (Ikuspegi, 2014) muestra que casi el $25 \%$ de la población vasca quiere vivir en un barrio donde nadie sea de una raza o grupo étnico distinto al de la mayoría de la población; el $27 \%$ considera que su relación con la población extranjera ha empeorado en el último año, frente al 14,6\% que lo pensaba en 2007; siete de cada diez personas consideran que, para aceptar plenamente a las personas inmigrantes, deberían abandonar las partes de su religión o cultura que entren en conflicto con nuestra legislación; tan sólo el $36,2 \%$ ve bien que ocupen plazas y espacios públicos; un $37 \%$, que construyan templos para sus religiones; y un $48,3 \%$, que hablen su propio idioma. También hay un $65 \%$ de personas que consideran que los inmigrantes se benefician en exceso del sistema de protección social y casi un $50 \%$, que pagan menos impuestos de los que luego reciben.

Sin embargo, la actitud de la población vasca hacia los inmigrantes se puede definir como ambivalente: se mezclan algunas posiciones cerradas con otras que abogan por equiparar derechos. El Observatorio Vasco de la Inmigración realiza un índice de tolerancia hacia la inmigración extranjera que resume las 
opiniones, actitudes, valores, creencias y estereotipos que se van desgranando a lo largo del barómetro. El cero sería el mayor grado de intolerancia y el cien, el mayor grado de tolerancia; en 2013, el índice global se situaba en 55,35 puntos, siendo 2012 el peor año, con 53,62 puntos, y 2008 el mejor, con 58,74 . El peor dato de la evolución de la actitud de la sociedad vasca hacia la inmigración es el del porcentaje de personas que, según el propio barómetro, se podría catalogar como reacias hacia la inmigración, que ha crecido del 19,3\% de 2008 al 30,2 \% de 2013, situándose el porcentaje de tolerantes en el 35,9\% y el de ambivalentes en el 33,9\%.

Viendo los datos del barómetro, ¿cómo podemos incidir en un cambio de actitudes de la población vasca hacia las personas inmigrantes? Son diversas las teorías que explican los prejuicios. La definición clásica es la de Allport (1979), para quien es “una actitud hostil o prevenida hacia una persona que pertenece a un grupo simplemente por el hecho de pertenecer a ese grupo, suponiéndose, por lo tanto, que posee las cualidades objetivables atribuidas a dicho grupo". Algunas teorías ponen el acento en las causas socioculturales, como las teorías del conflicto, que conciben el prejuicio como producto de una competición entre diferentes grupos debida a la escasez de recursos o a una amenaza que puede ser tanto real como simbólica, por ejemplo, costumbres y valores que se consideran tan diferentes que son una amenaza para la identidad propia (Stephan y Stephan, 2001). Otras teorías ponen el énfasis en las relaciones intergrupales, como la teoría de la identidad social (Tajfel y Turner, 1979); en este caso, el factor más importante en la creación de las actitudes y los estereotipos es la búsqueda de una identidad social positiva. Esta identidad es parte del autoconcepto de la persona, que deriva de su conocimiento de pertenencia a un grupo, y del valor y la significación emocional asociada a dicha pertenencia. Las citadas teorías explican el fenómeno de acentuación de las diferencias intercategoriales y las similitudes intracategoriales, del favoritismo intragrupal y de discriminación intergrupal. Por último, algunas teorías se centran en factores individuales, como los sesgos cognitivos (sesgo de confirmación, correlaciones ilusorias) o las atribuciones causales y los factores de personalidad, como la teoría de la orientación hacia la dominación social o la teoría de la personalidad autoritaria (Adorno, 1950).

La amplísima literatura en torno a los prejuicios debe servir para orientar las políticas que persiguen reducir o evitar la discriminación, el racismo y la xenofobia. A ello contribuyen también los diferentes modelos sobre la persuasión y el cambio de actitudes (Moscovici, 1985). Sin embargo, es necesario que las instituciones públicas aboguen decididamente por una estrategia clara de lucha contra la discriminación, que preste especial atención a las cuestiones que tienen que ver con programas de sensibilización, prevención y detección, y a las medidas de reparación a las víctimas de actos discriminatorios 0 racistas.
Por último, es necesario tomar en cuenta las nuevas formas que adquiere el racismo, el denominado neorracismo (Taguieff, 1995), consistente en:

- La traslación de la raza hacia la cultura, y la sustitución de la pureza racial por la identidad cultural auténtica.

- El desplazamiento de la desigualdad hacia la diferencia: el desprecio por las personas consideradas inferiores se complementa con la obsesión del contacto con los otros, con la fobia a la mezcla.

- El recurso a enunciados heterófilos (derecho a la diferencia) más que a enunciados heterófobos.

- El racismo simbólico o indirecto, expresado sin ser declarado, y que tiende a sustituir al racismo directo y declarado.

\subsection{Exclusión}

La exclusión social, para los grupos excluidos, equivale a la incapacidad de ejercer sus derechos sociales, con la imposibilidad consiguiente de una inserción e integración social plena (Laparra, Aguilar y Gaviria, 1995). Una de las definiciones de exclusión que más consenso generan alude a "un proceso de alejamiento progresivo de una situación de integración social en el que pueden distinguirse diversos estadios en función de la intensidad" (Fundación FOESSA, 2008: 184), "que incluye dificultades o barreras en al menos el eje económico (empleo, ingresos, privación), el eje político de la ciudadanía (los derechos políticos, pero también la educación, la salud o la vivienda) y el eje de las relaciones sociales (aislamiento, anomia)" (ibídem: 183).

La presencia desproporcionada de migrantes entre el colectivo de personas que sufren exclusión social ha sido ampliamente demostrada tanto a escala internacional (McLaren, 2003) como nacional. Los datos del VI Informe sobre exclusión y desarrollo social en España (Fundación FOESSA, 2008) muestran que la exclusión severa posee una sobrerrepresentación de nacidos fuera de España (31\%). La proporción de hogares afectados por procesos de exclusión en ámbitos como el empleo es del $\mathbf{2 7 , 2} \%$ en las personas extracomunitarias, frente a un $12,5 \%$ entre los españoles o comunitarios de la UE15. En la exclusión de la vivienda, las cifras son del $17,8 \%$ entre los segundos, frente al $37 \%$ entre de los primeros; y en el ámbito de la salud, del 10,8\% frente al 16,6\%. Es por ello por lo que el informe afirma que "la variable étnica aparece como la más determinante a la hora de distribuir socialmente las probabilidades de ser afectado por procesos de exclusión social” (ibídem: 244).

Quizá uno de los ejemplos más representativos es el de las personas sin hogar, que podemos considerar como el extremo de exclusión social. La primera gran encuesta sobre población sin hogar realizada en España por el INE en 2005 arrojaba una estimación de 21.900 personas sin hogar, de las cuales un 48,2 \% 
eran extranjeras. La segunda se realizó en 2012, y el número de personas sin hogar aumentó hasta las 22.938, de las cuales el 45,8\% eran extranjeras. La encuesta permite distinguir entre población sin casa (aquella que se encuentra alojada en algún recurso para personas sin hogar) y población sin techo (la que se encuentra durmiendo en la calle o en estructuras temporales). En el primer caso, las personas extranjeras suponían el $45 \%$ del total, y en el segundo, el $54 \%$ (INE, 2005, 2012). En la Comunidad Autónoma del País Vasco, se publicó un recuento nocturno realizado en las tres capitales vascas (SIIS, 2013), con datos aún más divergentes: el 76,9\% de las personas que dormían en la calle y el $60,3 \%$ de las que lo hacían en centros de acogida eran extranjeras.

La Ley de Servicios Sociales del Gobierno Vasco formulada en 2008 carece de un desarrollo normativo y una cartera de servicios definidos, lo cual ha impedido que la lucha contra la exclusión social avance de manera efectiva. En la actualidad, se cuenta con un borrador de la cartera de servicios, que parece será aprobado en el futuro, en el que se establece una clara distinción entre los servicios sociales de atención primaria, cuya competencia corresponde a los ayuntamientos, y los servicios sociales de atención secundaria, cuya competencia es de las diputaciones. Entre los primeros, entran los servicios de atención diurna (que incluye comedores sociales), los servicios de acogida nocturna, pisos de acogida y viviendas tuteladas. Los servicios de competencia foral serían los centros de día para atender necesidades de inclusión social, centros de acogida nocturna y centros residenciales. La mayor diferencia entre los servicios de los ayuntamientos y los de las diputaciones estriba en que, para los segundos, se necesita una valoración y derivación previa, al ser especializados, por lo que se deberá tener un cuidado especial para no dejar fuera a las personas migrantes por cuestiones administrativas o burocráticas, articulando las medidas necesarias para evitar una doble exclusión.

También es necesario actuar en la sociedad para evitar que las personas empobrecidas y excluidas sufran lo que algunos autores denominan deshumanización normativa (Reguera, 2007) o deslegitimación (Bar-Tal, 1990). Ambos conceptos aluden al proceso que degrada y deshumaniza a un individuo, incluyéndolo entre las categorías sociales más negativas, con el propósito de excluirlo de los grupos humanos que actúan dentro de los límites aceptables en términos de normas o valores. De esta manera, la despersonalización y la deshumanización tienen un gran poder autodesinhibidor que permite maltratar al otro sin experimentar reproche alguno. Aunque la mayoría de las personas rechazan la injusticia y piensan que les resulta difícil perjudicar a otros, sin embargo son capaces de hacerlo si las víctimas han sido previamente deshumanizadas y excluidas de la pertenencia a la “comunidad moral” (Opotow, 1990). Quizá el mejor ejemplo de ello son los denominados delitos de odio, de los que apenas se tienen conocimiento en el País Vasco, no tanto porque no ocurran, sino porque no se recogen como tales en los atestados policiales.

\subsection{Interacción}

Los datos expuestos anteriormente muestran los diferentes planteamientos existentes en torno al binomio clase-raza, o lo que es lo mismo, mientras que para unos la posición de inferioridad del inmigrante se debe a su pertenencia a una clase social desfavorecida, para otros la clave de la marginación se encuentra en el origen racial, étnico o religioso de los grupos inmigrados. El debate de fondo es la relación que se establece entre diferencia y desigualdad, si son las desigualdades las que crean las diferencias 0 viceversa.

Para los teóricos de orientación marxista-económica (Castles y Kosack, 1973; Zolberg, 1989), los trabajadores extranjeros se insertan en el estrato más bajo de la clase obrera, debido a la función que este tipo de mano de obra desempeña en el sistema capitalista mundial. La función del racismo, en este caso, es la de la legitimación ideológica de un proceso de dominación y explotación económica. En la lucha contra el racismo, se rechaza tomar en cuenta cualquier visión identitaria, al considerar que el fenómeno afecta a ciudadanos, a individuos, y no a grupos definidos por su diferencia étnica, cultural o religiosa.

Otros investigadores se preguntan si la posición social de determinados grupos étnicos en las sociedades de acogida es debida exclusivamente a la distinta cualificación del conjunto de sus miembros. El resultado es que a la estratificación de clase se le superpone la racial o étnica (Freeman, 1979). Argumentan que la discriminación no se debe a la falta de cualificación de los inmigrantes, esto es, a su condición de clase, sino a la diferencia de raza. Critican el planteamiento economicista, porque no da respuesta al neorracismo. Para las teorías más centradas en los aspectos identitarios, el núcleo duro del racismo es la naturalización de las diferencias. La clave reside en la pertenencia a una cultura, raza o etnia como elemento generador de relaciones sociales específicas. A ello se le une que la vida cotidiana de los individuos y grupos no se desarrolla tanto dentro de pautas de clase como de nuevas formas de identidad colectiva, que generan formas alternativas de solidaridad.

Quizá una posición intermedia nos la ofrece Goffman (1980) cuando describe el estigma. La estigmatización ocurre cuando una persona es negativamente evaluada, en términos de atribuciones desacreditantes, negativas, de ilegitimidad percibida o de una identidad social devaluada. Esta estigmatización conlleva una pérdida de estatus social y las personas con bajo estatus, al verse afectada la estructura que rodea al individuo y le protege de circunstancias desfavorables, son las que más sufren conductas de discriminación y exclusión. Además, frecuentemente se las considera un grupo frente al cual dichas conductas están justificadas.

En cualquier caso, la respuesta que demos a la interacción existente entre diferencia y desigualdad, 
marcará claramente las posibilidades de integración social en un futuro. Ello no quiere decir que se asimile inmigración con exclusión social, pero éste es un debate del que no podemos rehuir y que no parece estar en la agenda inmediata de las instituciones públicas, ni tan siquiera de las sociales, a pesar de que los datos aportados sobre exclusión social muestran claramente su incidencia en el colectivo migrante; y a pesar también de que la actitud de la población autóctona hacia la extranjera, si bien no resulta especialmente intolerante en el caso del País Vasco, no sólo no mejora con el paso del tiempo, sino que empeora ligeramente, tal y como hemos comprobado en el barómetro de Ikuspegi. Contamos con las condiciones adecuadas para que la integración de las personas migrantes sea vivida de forma positiva, pero también hay elementos preocupantes que deben abordarse con celeridad. Del éxito de la tarea depende, en gran medida, el futuro de la cohesión social del País Vasco.

\section{Bibliografía referenciada}

ACHOTEGUI, J. (2006): “Estrés límite y salud mental: el síndrome del inmigrante con estrés crónico y múltiple (síndrome de Ulises)", Migraciones, $\mathrm{n}$ 은 19 , págs. 59-85.

- (2002): La depresión de los inmigrantes: una perspectiva transcultural, Barcelona, Ediciones Mayo.

ADORNO, T. W.; LEVINSON, D. J.; y SANFORD, N. (1950): The Authoritarian Personality, Oxford, Harpers.

ALLEYNE, B. (2002): "An idea of community and its discontents: Towards a more reflexive sense of belonging in multicultural Britain", Ethnic and Racial Studies, vol. 25, no 4, págs. 607-627.

ALLPORT, G. W. (1979): The Nature of Prejudice, Cambridge, Addison-Wesley.

AYUNTAMIENTO DE BILBAO (2013): “Acta de la sesión ordinaria celebrada por el Excmo. Ayuntamiento. Pleno el día 25 de septiembre de 2013", págs. 16-36 [<http://www. nuevosvecinos.com/descargas/deustobarria/ acta_plen020130925.pdf〉.

BARCELONA CENTRE FOR INTERNATIONAL AFFAIRS; MIGRATION POLICY GROUP (2015): Migrant Integration Policy Index [rhttp://www.mipex. eus].

BAR-TAL, D. (1990): “Causes and consequences of delegitimization: Models of conflict and ethnocentrism", Journal of Social Issues, vol. $46, \mathrm{n} \stackrel{0}{1}$, págs. $65-81$.

BERRY, J. W. (2001): “A psychology of immigration”, Journal of Social Issues, vol. 57, nํ 3, págs. 615-631 [<http://dx.doi.org/10.1111/0022-4537.00231)].

BERRY, J. W.; y KIM, U. (1988): Acculturation and Mental Health. Health and Cross Cultural Psychology: Towards Applications, Londres, Sage.

BESS, K. D. et al. (2009): "Participatory organizational change in community-based health and human services: From tokenism to political engagement", American Journal of Community Psychology, vol. 43, no 1-2, págs. 134-148.

BETANCOURT, J. R. et al. (2003): “Defining cultural competence: A practical framework for addressing racial/ethnic disparities in health and health care", Public Health Reports, vol. 118, nํ4, págs. 293-302.

BLANCO, A.; y DÍAZ, D. (2006): "Orden social y salud mental: una aproximación desde el bienestar social”, Clínica y Salud, vol. 17, nº 1, págs. 7-29.

BLANCO, A.; y RODRÍGUEZ MARÍN, J. (2007): Intervención psicosocial, Madrid, Prentice-Hall.

BRONFENBRENNER, U. (1979): The Ecology of Human Development, Cambridge, Harvard University Press.

CACHÓN, L. (2009): La 'España inmigrante': marco discriminatorio, mercado de trabajo y políticas de integración, Barcelona, Anthropos.

- (2008): “La integración de y con los inmigrantes en España: debates teóricos, políticas y diversidad territorial”, Política y Sociedad, vol. 45, nํㅜ 1 , págs. 205-235.

CASTLES, S.; y KOSACK, G. (1973): Immigrant Workers and Class Structure in Western Europe, Londres, Oxford University Press.

DE LA MATA, M. L. et al. (2010): "La integración de las personas migrantes: el enfoque de la psicología cultural y de la liberación”, en MELERO VALDÉS, L. (coord.), La persona más allá de la migración. Manual de intervención psicosocial con personas migrantes, Valencia, Fundación CeiMigra, págs. 115-146.

DE LUCAS. J.; y SOLANES, A. S. (eds.) (2009): La igualdad en los derechos: claves de la integración, Madrid, Dykinson.

EAGLY, A. H.; y CHAIKEN, S. (1993): The Psychology of Attitudes, San Diego, Harcourt Brace Jovanovich. 
ESPAÑA (2000): “Ley Orgánica 4/2000, de 11 de enero, sobre Derechos y Libertades de los Extranjeros en España y su Integración Social”, Boletín Oficial del Estado, nํ10, 12-1-2000, págs. 1.139-1.150 [khttp://www.boe.es/buscar/doc. php?id=BOE-A-2000-544'].

FERNÁNDEZ LIRIA, A.; y PÉREZ-SALES, P. (2011): Instrumento para la Valoración de la Competencia Intercultural en la Atención en Salud Mental (IVCI), Madrid, Dirección General de Salud Pública y Sanidad Exterior del Ministerio de Sanidad, Política Social e Igualdad.

FREEMAN, G. P. (1979): Immigrant Labor and Racial Conflict in Industrial Societies: The French and British Experience, New Jersey, Princeton University Press.

FUNDACIÓN FOESSA (2008): VI Informe sobre Exclusión y Desarrollo Social en España, Madrid, Fundación FOESSA.

GARCÍA FUSTER, E.; HERRERO OLAIZOLA, H.; y MUSITU, G. (1995): El apoyo social, Barcelona, PPU.

GOBIERNO VASCO (2011): III Plan Vasco de Inmigración, Ciudadanía y Convivencia Intercultural 20112013, Vitoria-Gasteiz, Servicio Central de Publicaciones del Gobierno Vasco.

- (2007): II Plan Vasco de Inmigración, 2007-2009, VitoriaGasteiz, Servicio Central de Publicaciones del Gobierno Vasco.

- (2003): I Plan Vasco de Inmigración, 2003-2005, VitoriaGasteiz, Servicio Central de Publicaciones del Gobierno Vasco.

GODENAU, D. et al. (2014): La integración de los inmigrantes en España: una propuesta de medición a escala regional, serie Documentos del Observatorio Permanente de la Inmigración, no 30, Madrid, Observatorio Permanente de la Inmigración.

GOFFMAN, E. (1980): Estigma: la identidad deteriorada, Buenos Aires, Amorrortu.

HERNÁNDEZ PLAZA, S.; POZO MUÑOZ, C.; y ALONSO MORILLEJO, E. (2004): “Apoyo social y bienestar subjetivo en un colectivo de inmigrantes: ¿efectos directos o amortiguadores?", Boletín de Psicología, vol. 80, no 80, págs. 79-96.

IKUSPEGI (2014): Barómetro 2013. Percepciones y actitudes hacia la inmigración extranjera, Bilbao, Ikuspegi-Observatorio Vasco de Inmigración.

INE (2012): Encuesta sobre las Personas sin Hogar (Personas). Año 2012, Madrid, Instituto Nacional de Estadística.

- (2005): Encuesta sobre las personas sin hogar (personas). Año 2005, Madrid, Instituto Nacional de Estadística.

KEYES, C. (1998): “Social well-being”, Social Psychology Quarterly, vol. 61, nํ2, págs. 121-140.

LAPARRA, M.; AGUILAR, M.; y GAVIRIA, M. (1995): "Aproximación teórica al concepto de exclusión”, en ÁLVAREZ-URÍA, F. et al., Desigualdad y pobreza hoy, Talasa, págs. 133200.

MALGESINI, G.; y GIMÉNEZ, C. (2000): Guía de conceptos sobre migraciones, racismo e interculturalidad, Madrid, Libros de la Catarata.
MARKEZ-ALONSO, I.; FERNÁNDEZ-LIRIA, A.; y PÉREZ-SALES, P. (2009): Salud mental y violencias institucional, estructural, social y colectiva, Madrid, Asociación Española de Neuropsiquiatría.

MARTín-BARÓ, I. (2003): Poder, ideología y violencia, Madrid, Trotta.

MCLAREN, L. M. (2003): “Anti-immigrant prejudice in Europe: Contact, threat perception, and preferences for the exclusion of migrants", Social Forces, vol. 81, n- 3, págs. 909-936.

MOSCOVICI, S. (1985): Psicología social: influencia y cambio de actitudes: individuo y grupos, Barcelona, Paidós.

OPOTOW, S. (1990): “Moral exclusion and injustice: An introduction", Journal of Social Issues, vol. 46 , $\mathrm{n}$ 을. págs. 1-20.

PALOMA, V.; y MANZANO-ARRONDO, V. (2011): “El rol de las organizaciones desde la psicología de la liberación: aplicaciones en el estudio de las migraciones", Psychosocial Intervention, vol. 20, $\mathrm{n}^{0} 3$, págs. 1-11.

PAÍS VASCO (2012): "Decreto 114/2012, de 26 de junio, sobre Régimen de las Prestaciones Sanitarias del Sistema Nacional de Salud en el Ámbito de la Comunidad Autónoma de Euskadi”, Boletín Oficial del País Vasco, n- 248, 31-12-2013 [<http://www.euskadi.eus/r47-bopvvaci/es/ bopv2/datos/2013/12/1305620a.pdf>].

- (2010): "Decreto 147/2010, de 25 de mayo, de la Renta de Garantía de Ingresos", Boletín Oficial del País Vasco, no 114, 17-6-2010 ['http://www. lehendakaritza.ejgv.euskadi.net/r48-vacia/es/ bopv2/datos/2008/12/0807235a.pdf)].

- (2008): "Ley 12/2008, de 5 de diciembre, de Servicios Sociales", Boletín Oficial del País Vasco, nํ-246, 24-12-2008, págs. 31.84031.924 [khttp://www.euskadi.net/bopv2/ datos/2008/12/0807143a.pdf>]

- (2008): “Ley 18/2008, de 23 de diciembre, para la Garantía de Ingresos y para la Inclusión Social", Boletín Oficial del País Vasco, nํㅜ 250, 31-12-2008, págs. 32.729-32.804 [<http://www. lehendakaritza.ejgv.euskadi.net/r48-vacia/es/ bopv2/datos/2008/12/0807235a.pdf)].

PHINNEY, J. S. et al. (2001): "Ethnic identity, immigration, and wellbeing: An interactional perspective", Journal of Social Issues, vol. $57, \mathrm{n}-3$, págs. 493-510.

PRILLELTENSKY, I. (2012): “Wellness as fairness", American Journal of Community Psychology, vol. 49, n1-2, págs. 1-21 [khttp://dx.doi.org/10.1007/ s10464-011-9448-8>]

REGUERA, G. B. (2007): “La demonización del otro, la deshumanización y el racismo", Cuadernos del Ateneo de La Laguna, ํㅡㄴ 24, págs. 18-28.

SIIS CENTRO DE DOCUMENTACIÓN Y ESTUDIOS (2013): Estudio sobre la situación de las personas en situación de exclusión residencial grave en la $C A P V$, Vitoria-Gasteiz, Departamento de Empleo y Asuntos Sociales del Gobierno Vasco.

SPENCER, S.; y COOPER, B. (2007): Social Integration of Migrants in Europe: A Review of the European Literature 2000-2006, Oxford, Centre on Migration, Policy and Society. 
STEPHAN, W. G.; y STEPHAN, C. W. (2001): Improving Intergroup Relations, Thousand Oaks, Sage.

TAGUIEFF, P. A. (1995): "Las metamorfosis ideológicas del racismo y la crisis del antirracismo", en ALBITE, J. P. (coord.), Racismo, antirracismo e inmigración, Donostia, Gakoa, págs. 143-204.

TAJFEL, H.; y TURNER, J. C. (1979): “An integrative theory of intergroup conflict”, en AUSTIN, W. G.; y WORCHEL, S. (eds.), The Social Psychology of Intergroup Relations, Monterey, Brooks-Cole, págs. 33-47.

TASHAKKORI, A.; y TEDDLIE, C. (eds.) (2010): Handbook of Mixed Methods in Social \& Behavioral Research, Thousand Oaks, Sage.
TOMÁS-SÁBADO, J. et al. (2007): “Construction and preliminary validation of the Barcelona Immigration Stress Scale", Psychological Reports, vol. 100, nํㅜ 3, págs. 1.013-1.023.

VILLASANTE, T. R. (1984): Comunidades locales. Análisis, movimientos sociales y alternativas, Madrid, Instituto de Estudios de la Administración Local.

ZOLBERG, A. R. (1989): "The next waves: Migration theory for a changing world", International Migration Review, vol. 23, nํㅜ 3, págs. 403-430. 